\title{
Die Einarbeitung postkolonialer Perspektiven in die Arbeit mit Freiwilligen und Partnerorganisationen: Ein Projekt der Volunteers' Abroad Programs des Internationalen Bundes (IB VAP)
}

\author{
Nils Witt \\ B.A. in Regionalstudien Lateinamerika | freiberuflicher Trainer, \\ Mediator und Projektmanager | nils.witt@posteo.de
}

Der Internationale Bund (IB) mit Hauptsitz in Frankfurt a. M. ist eine Entsendeorganisation für internationale Freiwilligendienste, die über die Volunteers ' Abroad Programs IB VAP mit Sitz in Fürth und Kassel Freiwillige ins Ausland entsenden. Seit dem Jahr 2004 werden über die IB VAP im Rahmen der herkömmlichen Programme (v. a. IJFD und weltwärts) Einsätze für einen in der Regel einjährigen Freiwilligendienst in Länder aller Kontinente vermittelt. Aktuell absolvieren 170 Freiwillige ihren Dienst.

Im Frühjahr 2013 trafen die Programmverantwortlichen der IB VAP die Entschei-

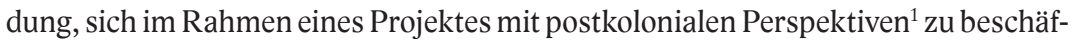
tigen und diese in das Programm zu integrieren. Die Beweggründe hierfür ergaben sich u. a. aus der Rückmeldung der Partnerorganisationen (PO) im Ausland zur Haltung der Freiwilligen, u. a. zur Motivation für einen Freiwilligendienst und bezüglich Vorstellungen über einen Freiwilligendienst, das Gastland und die eigene Rolle in der Aufnahmeorganisation. Auch den pädagogischen Mitarbeiter_innen beim IB ist in der Evaluierung der Begleitung von Freiwilligen aufgefallen, dass einige Freiwillige bezüglich postkolonialer Perspektiven über ihre Denk- und Verhaltensmuster noch nicht in letzter Konsequenz reflektieren und damit nicht nur die PO, sondern auch sich selbst emotional belasten. Solch bedenkenswerte Muster sind vor allem in der Vorbereitung vor der Ausreise ins Ausland, aber auch teilweise noch auf den Rückkehrseminaren wahrnehmbar. Dabei geht es vornehmlich um die Freiwilligen, deren Aufnahmeorganisationen im Globalen Süden verortet sind.

Zum Ausdruck kommt dies beispielsweise in einer Haltung bezüglich des Gefühls von vermeintlicher eigener Überlegenheit im Vergleich zu den Menschen im Globalen Süden (Superioritätskomplex). Bei Rückgekehrten kamen Fragen auf wie z. B.: Wieso konnte ich nicht so viel helfen?, Mir wurde ja mehr geholfen!, Müssen wir nicht mehr ändern?, Wieso wurde ich als Weiße_r so ungerecht behandelt?, Wieso wurden meine sinnvollen Vorschläge nicht umgesetzt? Nicht selten war zudem die

Postkoloniale Perspektiven oder die postkoloniale Theorie untersuchen die Wirkungen der Kolonialzeit auf die Gegenwart und den fortwährenden Einfluss kolonialer Strukturen auf das Denken und Handeln der Menschen und Gesellschaften im Globalen Norden und Süden. Dabei geht es um strukturell privilegierte und deprivilegierte Positionen im globalen Machtgefüge und daraus folgenden Diskriminierungen. 
Berichterstattung etwa in Blogs von einigen aktiven Freiwilligen und Rückgekehrten diskriminierend gegenüber den Menschen vor Ort. Auch in den Vorbereitungsseminaren der IB VAP, insbesondere in Einheiten zur Motivation für einen Freiwilligendienst im Ausland, sind die Pädagog_innen immer wieder mit Haltungen konfrontiert, die den Mitarbeiter_innen der PO als kompetent handelnden Individuen nicht gerecht werden.

Im Bewusstsein dieser anhaltenden Problematik wuchs das Bedürfnis bei den IB VAP, einen noch fachgerechteren Umgang mit Freiwilligen v.a. im Kontext der Nord-Süd-Freiwilligendienste zu erlernen.

Die Beschäftigung mit der Broschüre „Mit kolonialen Grüßen...“ des Berliner Vereins für machtkritische Bildungsarbeit glokal e. V. ${ }^{2}$ sowie unterschiedliche Erfahrungen von IB-Mitarbeiter_innen führten zu dem Entschluss, sich intensiv mit der postkolonialen Theorie und ihrer Bedeutung für die Arbeit der IB VAP zu beschäftigen. Eine solche Auseinandersetzung kann sich jedoch nicht nur auf die pädagogische Arbeit der EO beschränken. Dies bedeutet vielmehr, sich auch mit der eigenen Verortung im historischen und programmatischen Kontext von internationalen Freiwilligendiensten, mit der Zusammenarbeit mit den PO und nicht zuletzt mit der jeweils eigenen Haltung zu beschäftigen. Im Rahmen einer weltwärts-Begleitmaßnahme wurde der Prozess von glokal e.V. kompetent begleitet.

Das Projekt begann im ersten Schritt mit einem dreitägigen Inhouse-Workshop, geleitet von zwei Referenten von glokal e.V., an welchem Hauptamtliche sowie Honorar-Teamer_innen der IB VAP teilnahmen. Nach einem Input zu postkolonialer Theorie und einem historischen Rückblick ging es in den Workshop-Phasen darum, die eigene Arbeit durch eine postkoloniale Brille zu durchleuchten und an gegebener Stelle zu hinterfragen. Der inhaltliche Rahmen wurde dann auf eine generell machtkritische Analyse erweitert und durch einen inhaltlichen Block zu Organisationsentwicklung abgerundet. Immer wieder wurden auch Methoden aus dem Anti-Bias-Ansatz angewandt, die in die Arbeit mit den Freiwilligen aufgenommen werden können.

Im Anschluss an den Workshop wurden verschiedene Seminareinheiten, Bildungsmaterialien sowie Inhalte der Öffentlichkeitsarbeit überarbeitet. Zudem wurde basierend auf den Ergebnissen des Workshops Handlungsempfehlungen für die Optimierung der Zusammenarbeit mit PO, zur Diversifizierung der Zielgruppe, zum Umgang mit Incoming-Freiwilligendiensten und zur weiteren Auseinandersetzung mit postkolonialer Theorie erarbeitet.

2 Die Broschüre gibt es kostenlos zum Download unter: http://www.glokal.org/publikationen/mit-kolonialengruessen/ 
Die Auseinandersetzung mit postkolonialer Theorie und der dahingehenden Durchleuchtung der eigenen Arbeit im Kontext von Nord-Süd-Freiwilligendiensten ist ein sehr intensiver Prozess. Dieser wurde mit ersten Reflektionen und Ergebnissen begonnen: Im Bildungsbereich sollen beispielweise angehende Freiwillige in der Vorbereitung dazu angeregt werden, sich ihrer relativ privilegierten Position bewusst zu werden. Dies kann exemplarisch durch einen Reflektionsprozess über den Sinn von internationalen Freiwilligendiensten oder deren internationale Beschränktheit (Ungleichgewicht und Einschränkungen beim IncomingProgramm) geschehen. Ebenfalls gilt es, eine kritische Auseinandersetzung mit Konzepten von „Entwicklung“ anzustoßen. Dabei ist es den Mitarbeiter_innen der IB VAP wichtig, dass sich Freiwillige als Lernende begreifen, die in ihrer Aufnahmeorganisation unterstützend aktiv sind. Die Aufnahmeorganisationen sollen von allen Beteiligten als handelnde, kompetente Institutionen wahrgenommen und auch in der Öffentlichkeitsarbeit entsprechend dargestellt werden. Ihnen soll beispielsweise in Projektmappen, aber auch auf der Homepage die Möglichkeit gegeben werden, sich selbst in Form von Berichten oder Videos darzustellen, anstatt dass vornehmlich über sie berichtet wird. In der Zusammenarbeit mit PO wird zukünftig noch genauer geklärt, wie Aufnahmeorganisationen im Globalen Süden noch mehr Mitbestimmung bei der Gestaltung der Programme bekommen können, über die Auswahl der Freiwilligen und die Gestaltung von Seminaren sowie der pädagogischen Begleitung und der Anleitung in der Einsatzstelle hinaus.

Zudem gilt es, sich auch weiteren Fragen zu stellen, z. B.: Inwieweit wird es gelingen, eine diversitätsbewusste, rassismuskritische Haltung sowohl bei den Freiwilligen als auch bei den Partner_innen und bei den IB-Mitarbeiter_innen selber nachhaltig zu etablieren? Können (Incoming-)Freiwilligendienste so gestaltet werden, dass sie rassismuskritischen Kriterien standhalten können? Und was ist dabei in dem vorgegebenen Rahmen beispielweise von weltwärts überhaupt möglich? Inwieweit können, wollen und müssen sich alle Beteiligten verändern und weiterentwickeln?

Diese Fragen verdeutlichen, dass die Auseinandersetzung mit postkolonialer Theorie und Machtkritik die Bereitschaft erfordern, die eigene „Komfortzone“ zu verlassen um mit Inhalten konfrontiert zu werden, die womöglich noch unbekannt waren oder die Unwohlsein oder Unsicherheit entstehen lassen können. Die Thematik ist sehr komplex, und oft liegen historisch bedingte, ungewollte rassifizierte Denk- und Handlungsmuster in der Arbeit mit PO und auch mit Freiwilligen im Unbewussten.

Die Erfahrung aus diesem Projekt zeigt, dass es wichtig ist, alle Beteiligten auf einen Wissensstand zu bringen und einen Veränderungsprozess so partizipativ wie möglich zu gestalten. Hierzu ist eine wichtige Voraussetzung, Strukturen für Auseinandersetzungen, Austausch und Reflektion zu schaffen. Die Frage, wie weit 
ein solcher Prozess unter wessen Beteiligung gehen kann, ist dabei wohl die grundlegendste. Nach der Sicherung erster Ergebnisse sind die IB VAP weiterhin mit dieser und den vorangegangenen Fragen im Rahmen eines andauernden Prozesses beschäftigt.

Letztlich ist es für Träger internationaler Freiwilligendienste essentiell, die Verantwortung einer ganzheitlichen Betrachtung der eigenen Positionierung, Verhaltens- und Denkmuster unter dem Fokus auf die postkoloniale Prägung des Arbeitsfeldes der Nord-Süd-Freiwilligendienste anzunehmen. Eine Auseinandersetzung mit postkolonialer Theorie ist dabei ein wichtiger Schritt auf dem Weg zu einer diskriminierungsfreieren Arbeit.

Für Nachfragen zu dem Projekt oder bei Interesse an der Umsetzung eines ähnlichen Projektes steht der Autor dieses Beitrages gerne zur Verfügung. 